\title{
A Hybrid Framework for Adaptive Protection of Microgrids Based on IEC 61850
}

\author{
Akhtar Hussain and Hak-Man Kim* \\ Incheon National University, Korea. \\ hmkim@inu.ac.kr
}

\begin{abstract}
Microgrids can operate both in grid-connected and islanded modes. It is essential to protect microgrids against all types of faults in both of the operation modes. Conventional overcurrent protection schemes are not adequate for microgrids due to bidirectional flow of fault current and limited flow of fault current through semiconductor devices. In addition, the setting values of all the concerned relays need to be updated after any change in system operating conditions. Centralized and decentralized adaptive protection schemes are generally used for protection of microgrids. In this paper, a hybrid adaptive protection scheme has been proposed for protection of microgrids. Computational burden and data storage is distributed among the local controllers and the central controller. A gateway is proposed for communicating between the serial interfaced devices and the IEC 61850 process bus. Finally, a framework has been introduced for implementing the proposed hybrid protection scheme for adaptive protection of microgrids by using IEC 61850-based intelligent electronic devices (IEDs).
\end{abstract}

Keywords: Adaptive protection, campus microgrid, hybrid protection, IEC 61850, microgrid protection, intelligent electronic devices

\section{Introduction}

Achievement of sustainable energy is one of the major challenges for modern societies. Adoption of renewable and sustainable energies in buildings and institutional campuses can contribute to energy conservation [1]. Those university campuses, which have both distributed energy resources and local loads, are known as campus microgrids. The objective of campus microgrids is to aggregate existing on-site generations with multiple loads which are co-located in the institution or campus. Campus microgrids have attracted the attention of researchers due to single ownership of both generations and loads, and all the resources are located within a tight geography [2]. These traits of campus microgrids avoid many of the regulatory problems as compared to other types of microgrids. The benefits of campus microgrids are same with those of other microgrid categories, i.e. ability to mitigate power disruption economic impacts [3], ability to operate in both gridconnected and islanded modes [4], service reliability in islanded mode operation [5], etc.

In order to achieve the above-mentioned benefits from microgrids, several technical issues need to be addressed. One of the major challenges is a protection system for microgrids, which can respond to all types of faults in microgrids [6]. On one hand, the protection system should be capable of isolating the microgrid in case of main grid side faults. On the other hand, it should isolate minimum area of microgrid in case of microgrid faults. The protection

system for microgrids should be capable of handling the issues related to bidirectional flow of fault current [7] and limited flow of fault current through silicon devices [8]. Inverter fault currents are limited by the ratings of the devices to around 2 p.u. rated

* Corresponding Author 
current [8]. Therefore, conventional overcurrent protection techniques may not be adequate for microgrids.

In order to overcome the limitations of conventional over-current protection schemes, various other protection methodologies have been explored. A voltage-based protection scheme has been proposed by [9] for protection of microgrids. Voltages of micro-sources have been monitored and transformed to DC quantities through dq-transformation. The disturbance in the d-q values have been used for detection of faults in microgrids. Voltage dips have been used for detecting faults in microgrids by [10]. It has been observed that the depth of voltage dip depends on distance from the fault and fault resistance. Angle difference between zero and positive sequence currents have been used by [11] for generating fault indicator (FI) in feeder remote terminal units (FRTUs). An N-version protection methodology for microgrids has been proposed by [12]. The proposed protection methodology ensures accurate fault detection in microgrids through redundancy of protection algorithms.

In order to anticipate the impact of change in microgrid configuration and to change the settings of relays accordingly, adaptive protection has been employed by several researchers. In such schemes, communication network plays a key role. The protective devices need to communicate to locate and isolate the fault [13]. A communicated-based protection scheme has been proposed by [14] for isolated microgrids. A data mining approach has been used for identifying the setting values and other parameters of relays. A centralized protection methodology for protection of distributed energy resources (DERs) has been proposed by [15]. A framework has been modeled for implementing the proposed protection scheme through IEC 61850-7-420. The protection principles for microgrid in accordance to the IEC 61850 standard are summarized by [16].

The adaptive protection schemes available in the literature are either centralized or decentralized. The centralized architectures are simpler because the local devices do not take decisions. However, the computational burden of the central controller increases with the increase in network size [6]. In decentralized architecture, computational burden is shared among the local controllers but each local controller has to take actions independently, which requires computationally sound local controllers [6]. A hybrid adaptive protection scheme may exploit the merits of both centralized and decentralized adaptive methods. Similarly, the hybrid adaptive scheme may overcome the demerits of each of the commonly practiced (centralized and decentralized) adaptive protection schemes. In addition, due to layered data processing (process, bay, and station levels) in IEC 61850 , hybrid adaptive protection system could be easily implemented by using IEC 61850-based intelligent electronic devices (IEDs).

In this paper, a hybrid protection scheme has been presented for adaptive protection of microgrids. The station level devices are functioning as central controller while the process level devices are functioning as local controllers. Bay level IEDs are acting as a communication link between the station and process level devices. The computational burden and data storage is shared among the local controllers and the central controller. Local controllers can communicate among themselves and with the central controller through a common communication network. The end-devices can communicate with the process level IEDs through a serial communication link. A gateway has been introduced for transforming the serial data format to IEC 61850-based standard format and vice versa. Finally, a framework has been introduced for implementing the proposed hybrid protection scheme for adaptive protection of microgrids by using IEC 61850-based IEDs.

\section{Faults in Microgrids}

A typical microgrid model of IEC 61850-7-420 is shown in Figure 1(a). Generally, the faults of microgrids are divided into two major types depending on the operating state of the microgrid. The sensitivity, selectivity, and speed requirements for each fault group are 
different. Details about the requirement for each type of fault can be found in [6]. In grid connected mode, if any fault is observed in the external network (utility grid side), the static switch at point of common coupling (PCC) will be opened to isolate the microgrid from the main grid. In this way microgrid will operate autonomously.

A fault has been assumed in utility grid side of Figure 1(a) and is named as F1 as depicted in Figure 1(b). Fault current will flow from both the main feeder side and the microgrid side. The fault current from the microgrid side is the collective fault current of the entire active and connected distributed generators in the microgrid. Static switch of PCC will be opened after detecting the fault current. Due to opening of PCC microgrid will be protected from the fault current and will operate autonomously. Similarly, circuit breakers (CBs) across the fault will be opened to isolate the faulty section as shown in Figure 1(b).

Another fault F2 has been assumed in the microgrid side in grid-connected mode. Fault current from the main feeder side will combine with fault currents from DG1 and DG2 and flow in the downward direction. Fault current from DG3 will flow in the upward direction (opposite direction). Due to flow of fault current through PCC, the switch will open again to isolate the microgrid. In the meantime, CB5, CB8, and CB10 will be opened to isolate the faulty section. When the fault is cleared, PCC will be closed to acquire the previous state of microgrid. However, load3 will remain unserved until F2 is cleared.

If F2 is assumed in the microgrid system of Figure 1(a) for islanded mode, there will be no fault current from the main feeder side. However, the direction and flow of fault currents inside the microgrid will remain the same as depicted in Figure 1(b). In this case also, CB5, CB8, and CB10 will be opened to isolate the faulty section. Due to opening of the CBs surrounding load3, load3 will be disconnected from the power supply. The magnitude of fault current in the microgrid is different for grid-connected and islanded modes. Similarly, the direction of fault current could be different for faults in different locations. Therefore, different setting values of relays are required for each case to avoid malfunctioning of relays.
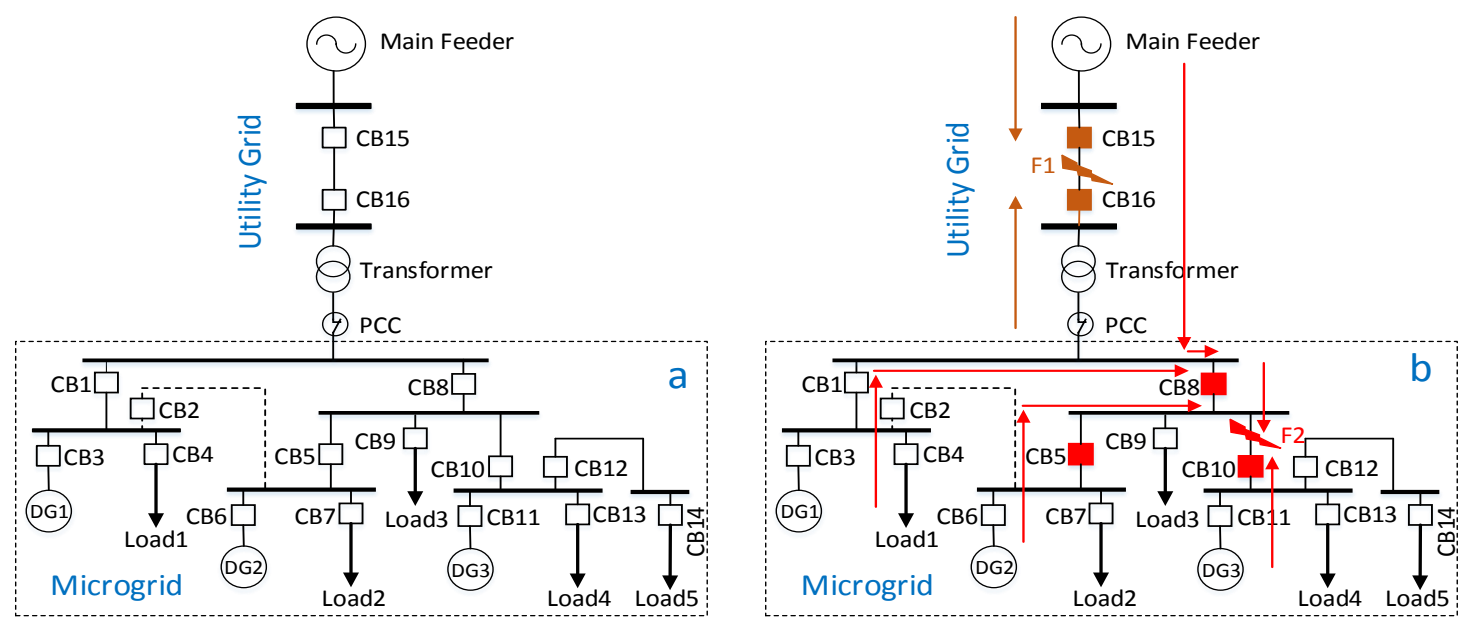

Figure 1. (a) Microgrid System Model According to IEC 61850-7-420 [15], (b) Fault Scenarios in Microgrids [6]

\section{Adaptive Protection Schemes for Microgrids}

\subsection{Centralized and Decentralized Adaptive Protection Schemes}

Adaptive protection is used for modifying the preferred protective response to any change in the condition or requirement of microgrid systems. This can be achieved either 
by means of externally generated signals or through control actions [6]. In order to realize an adaptive protection scheme for microgrids, digital relays and communication infrastructure are required. Digitals relays should have several setting values along with capability to sense current direction. Centralized and decentralized protection schemes are the two major types of adaptive protection schemes available in the literature.

An example of a centralized adaptive protection scheme for microgrids is shown in Figure 2. All the local controllers send their status information to the central controller. This is a master-slave scheme, where the central controller acts as a master and all the local controllers behave as slaves. Each local controller is responsible for informing the central controller about the occurrence of events within its locality. In addition, the central controller may periodically get the status information from each local controller. If any change is detected in the configuration of network, or status of end devices (DGs and loads), central controller will calculate the new settings for all the relays and inform all the respective local controllers. Each of the local controller has to follow the commands from the central controller.

An example of a decentralized adaptive protection scheme for microgrids is shown in Figure 3. In decentralized protection scheme, microgrid system is divided into small areas and is controlled by a local controller. Each local controller may communicate with its adjacent local controllers to find the direction of fault current and to isolate the faulty sections. Each local controller is equipped with the necessary intelligence and information to react upon any contingency in the system. A decentralized adaptive protection scheme needs to be implemented over a bus of an Ethernet network to realize the communication between all the local controllers. However, centralized adaptive protection schemes could be deployed with any type of communication, i.e. serial communication, bus communication, Ethernet network, or any other point-to-point communication.

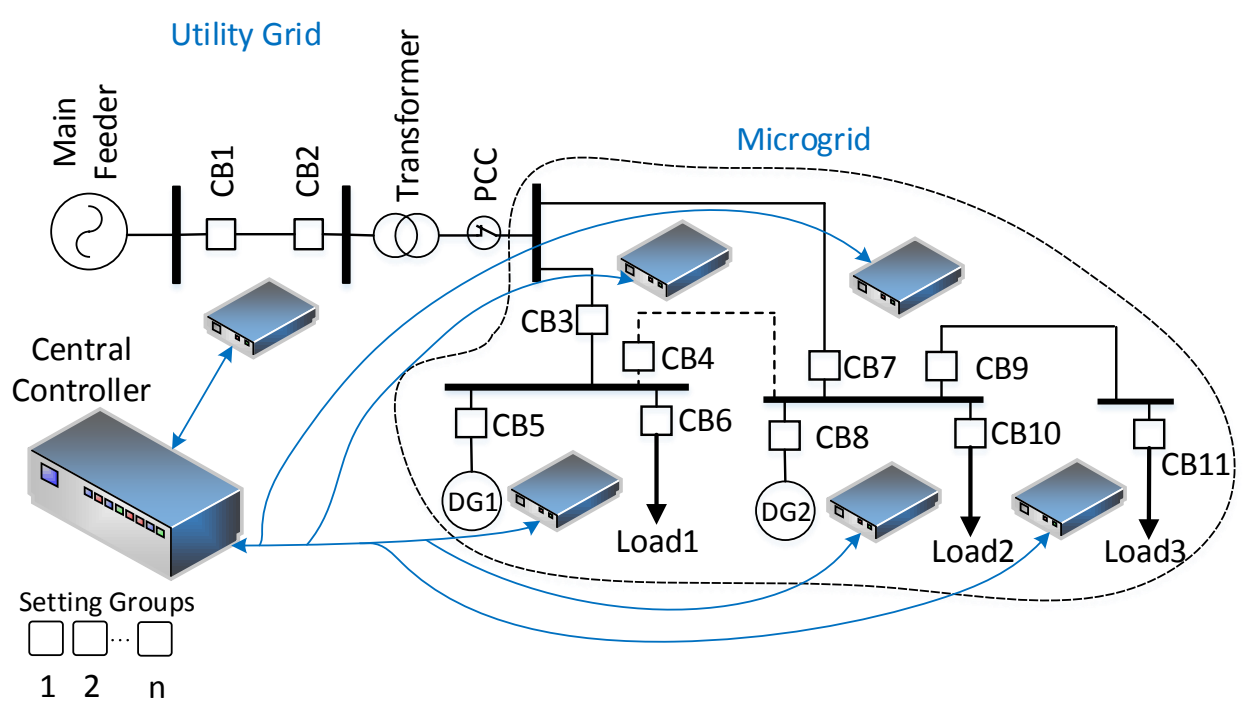

Figure 2. Centralized Adaptive Protection Scheme for Microgrids [17] 


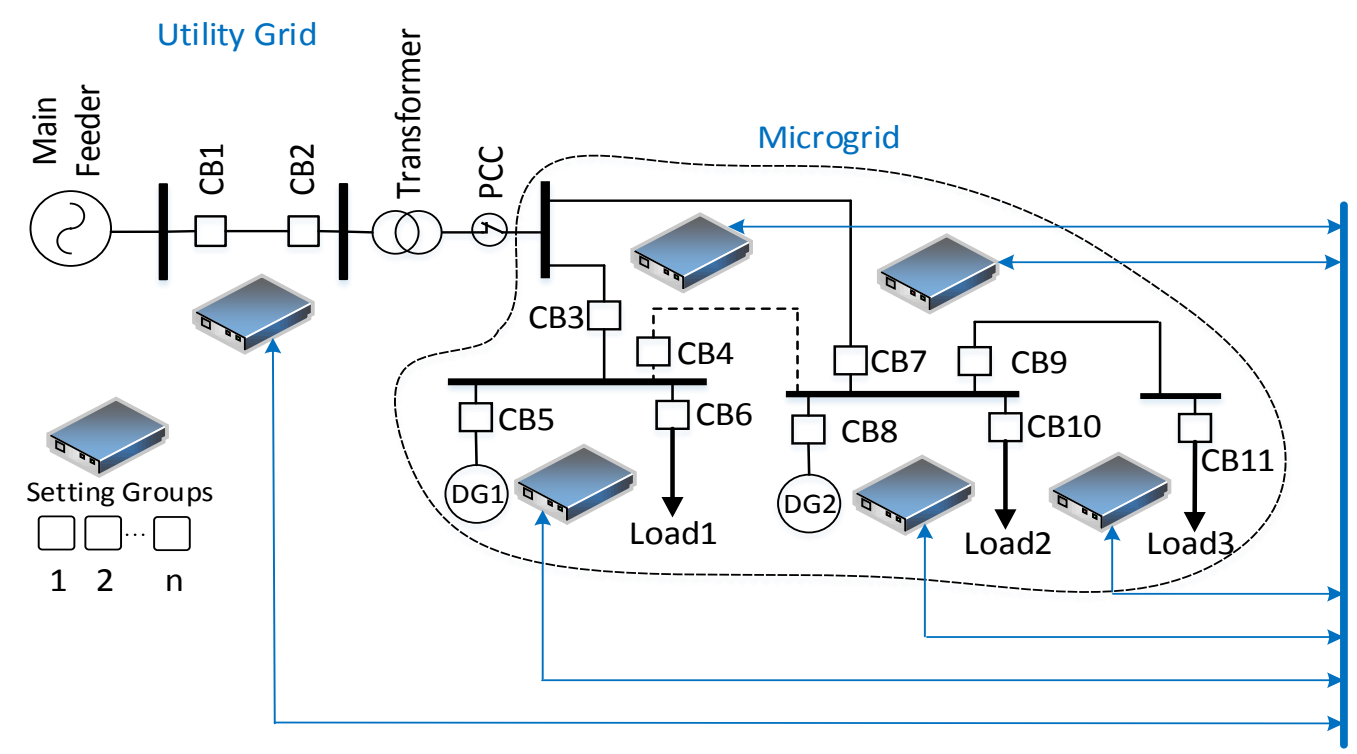

Figure 3. Decentralized Adaptive Protection Scheme for Microgrids [17]

\subsection{Proposed Hybrid Adaptive Protection Scheme}

In case of centralized adaptive protection schemes, the computational burden on the central controller increases with increase in the network size. In order to realize an adaptive protection scheme for microgrids, the central control need to do various data processing and has to record the events for each fault. The various types of data could be related to network configuration, listing of fault events, grid synchronization, interlocking logic, setting value groups, and logic for calculating new setting values with change in operating status of microgrid resources. While, in case of decentralized adaptive protection schemes, each local controller is equipped with all these capabilities.

A hybrid framework as shown in Figure 4 is proposed for adaptive protection of microgrids in this paper. Similar to the restoration technique proposed in [18] for smart distribution system, the computational burden is shared among the central and the local controllers. The proposed hybrid protection scheme is well suited for realization through IEC 61850 due to layered (process, bay, and station level) data processing in the IEC standard. Similarly, the storage of data is also distributed between the central and local controller as depicted in Figure 4. The responsibilities of the central and local controllers in the proposed hybrid adaptive protection scheme are as follows.

- The network configuration will be stored in the central controller. If any change is detected, the responsible local controller(s) will send the information to the central controller. The setting groups, which are calculated offline, will be stored in each local controller. Central controller will possess only an indexing table for each setting group. When any change in network configuration is reported, central controller will inform each local controller about the index of setting group and local controllers will adapt their relays to the new setting values.

- After the occurrence of an event, the event record will be stored in the respective local controller. Responsible local controller will send the timestamp with its id to the central controller. Central controller will only maintain a table listing the timestamp with local controller id. If the fault data is requested in a later stage, central controller may request the local controller. 


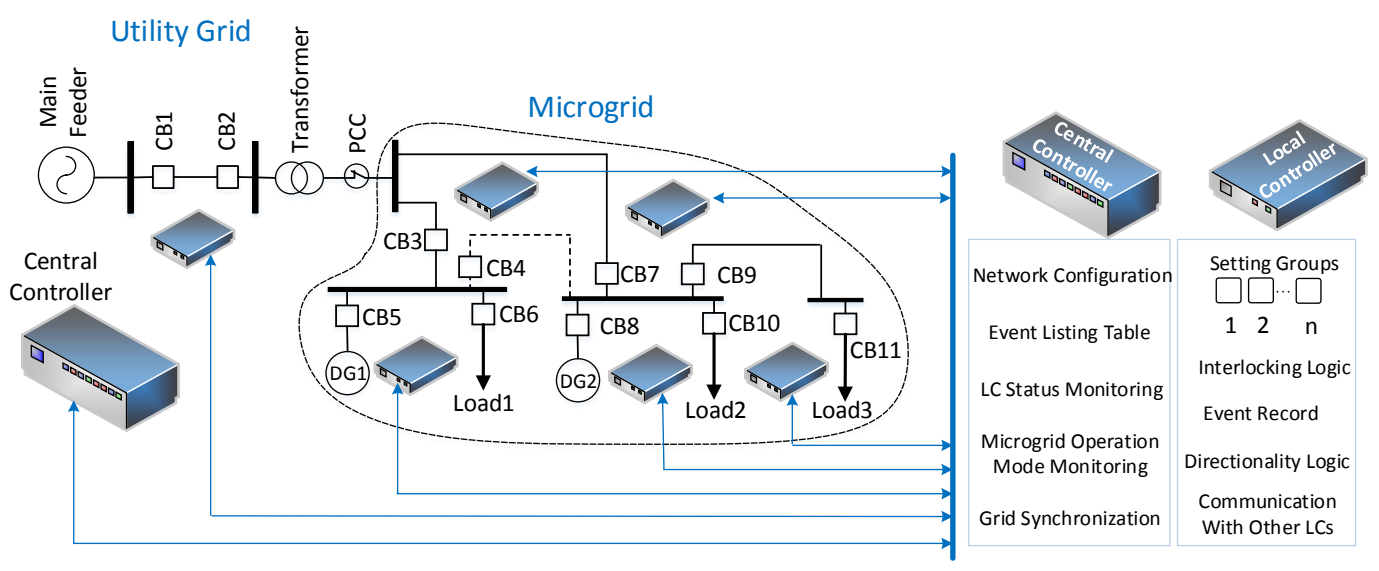

Figure 4. Proposed Hybrid Adaptive Protection Scheme for Microgrids

- The central controller will be responsible for monitoring the status of local controllers. Central controller may poll periodically to assess the status of local controllers. If any anomaly is detected by a local controller, it will inform the central controller.

- The operation mode of microgrid will be monitored by the central controller. Switching from grid-connected to islanded mode or vice-versa will be reported to each local controller. Each local controller will update the setting values of its relays according to the existing setting groups.

- The central controller is also responsible for receiving signals from the global positioning system (GPS) and for synchronizing all the local controllers. The local controllers will use that information to synchronize their local resources.

- The interlocking logic and logic for detecting the direction of fault current will be embedded in each of the local controller. In addition, all the local controllers are capable of communicating with each other and with the central controller through a common communication network.

\section{Realization through IEC 61850}

The objective of substation automation is to control, protect, and monitor the substation. IEC 61850 has evolved as a standard for substation automation. IEC 61850 enables the abstract definition of data items and services by using object-oriented hierarchical data modeling approach [19]. Generic object oriented substation event (GOOSE) messages are used for transmission of speedy and time-critical messages like tripping signals, status change, and blockings. Sampled values (SVs) are used for quickly transmitting synchronized current and voltage sampled values. In addition, COMTRADE files are used for recording the event data. IEC 61850 guarantees interoperability between intelligent electronic devices (IEDs) from different vendors, free architecture, long-term stability, and engineering based on substation configuration language (SCL) files [20].

\subsection{Component modeling in IEC 61850}

In order to assure interoperability, standardization of both the data objects and access to them is required. The standardized common services in IEC 61850 are reading data, writing data, controlling of devices, reporting of events, logging of events, and get directory. A hierarchical data model for a circuit breaker is shown in Figure 5. 


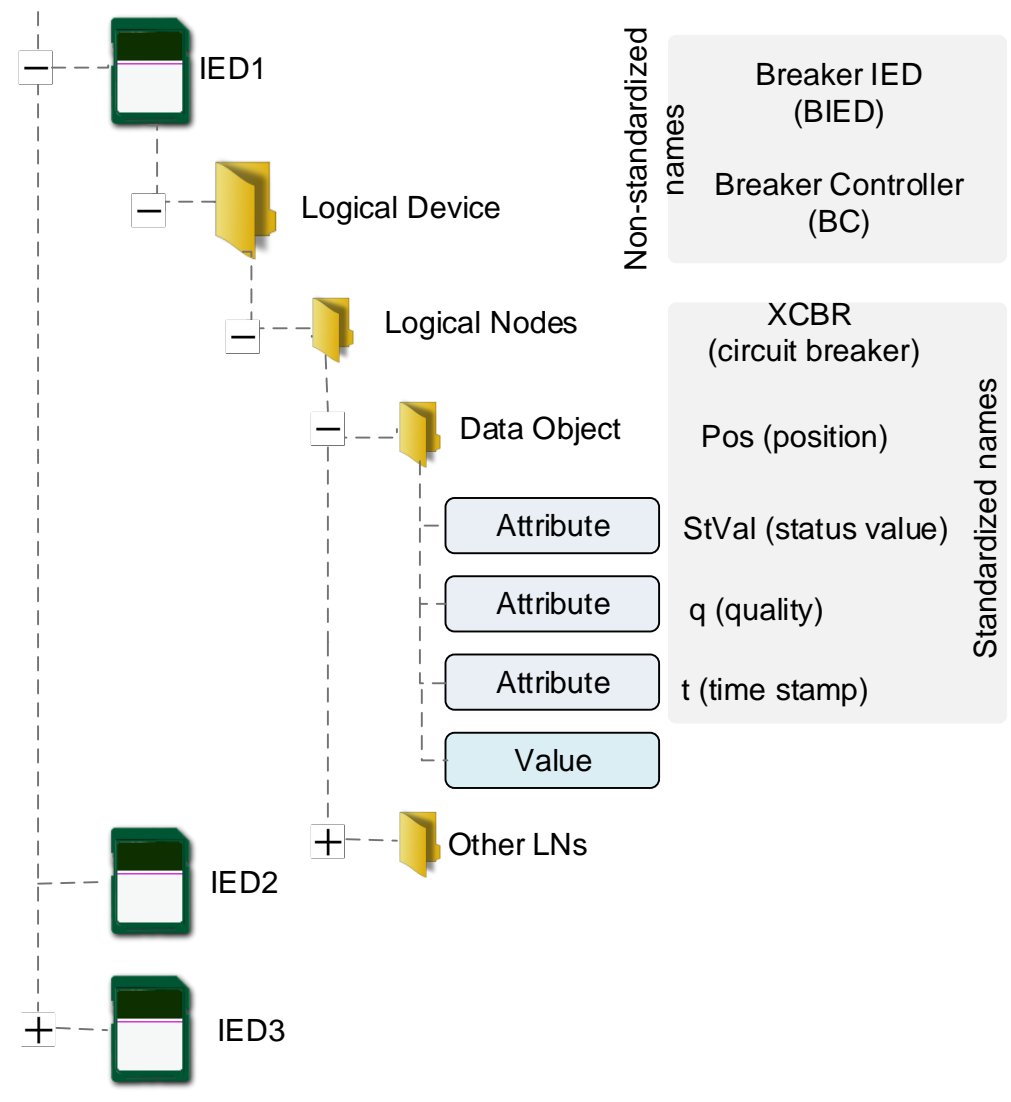

Figure 5. Hirerchial Data Model in IEC 61850 [20]

In order to implement the application functions in dedicated IEDs and to assure communication among them, application functions are broken down to smallest feasible pieces [19]. These basic objects are known as logical nodes (LNs) in IEC 61850 standard. The class name of the logical nodes refers to the function of the data objects (DOs), which belongs to it. The DOs contained in a LN may be mandatory, conditional, or optional. The DOs themselves contain attributes, which may be seen as values or detailed properties of the data objects. The names of LNs, DOs, attributes, and values are standardized in the standard. While the names of logical devices (LDs) and IEDs are not standardized [20].

\subsection{IEC 61850-7-420 and Microgrids}

Originally, the focus of IEC 61850 was on substation automation. Later it has been noticed that vendor-specific communication technologies impose major technical difficulties in other parts of the power system also, especially in the integration of DER technologies. A standard along with associated guidelines would simplify installation and maintenance costs, and improve reliability of the power system operation. Therefore, the scope of IEC 61850 has been revised and extended in the second edition to cover the entire power system [21]. A new part, 61850-7-420, has been included in the extended version in order to define LNs for DERs. Wind generation related logical nodes have been separately defined in IEC 61400-25-3 and object models for DER inverters have been defined in IEC 61850-90-7 [22]. The infor-mation exchange services have been defined in IEC 61850-7-2, which includes client-server abstract communication service interface (ACSI) services, GOOSE messages, and SVs [23]. 


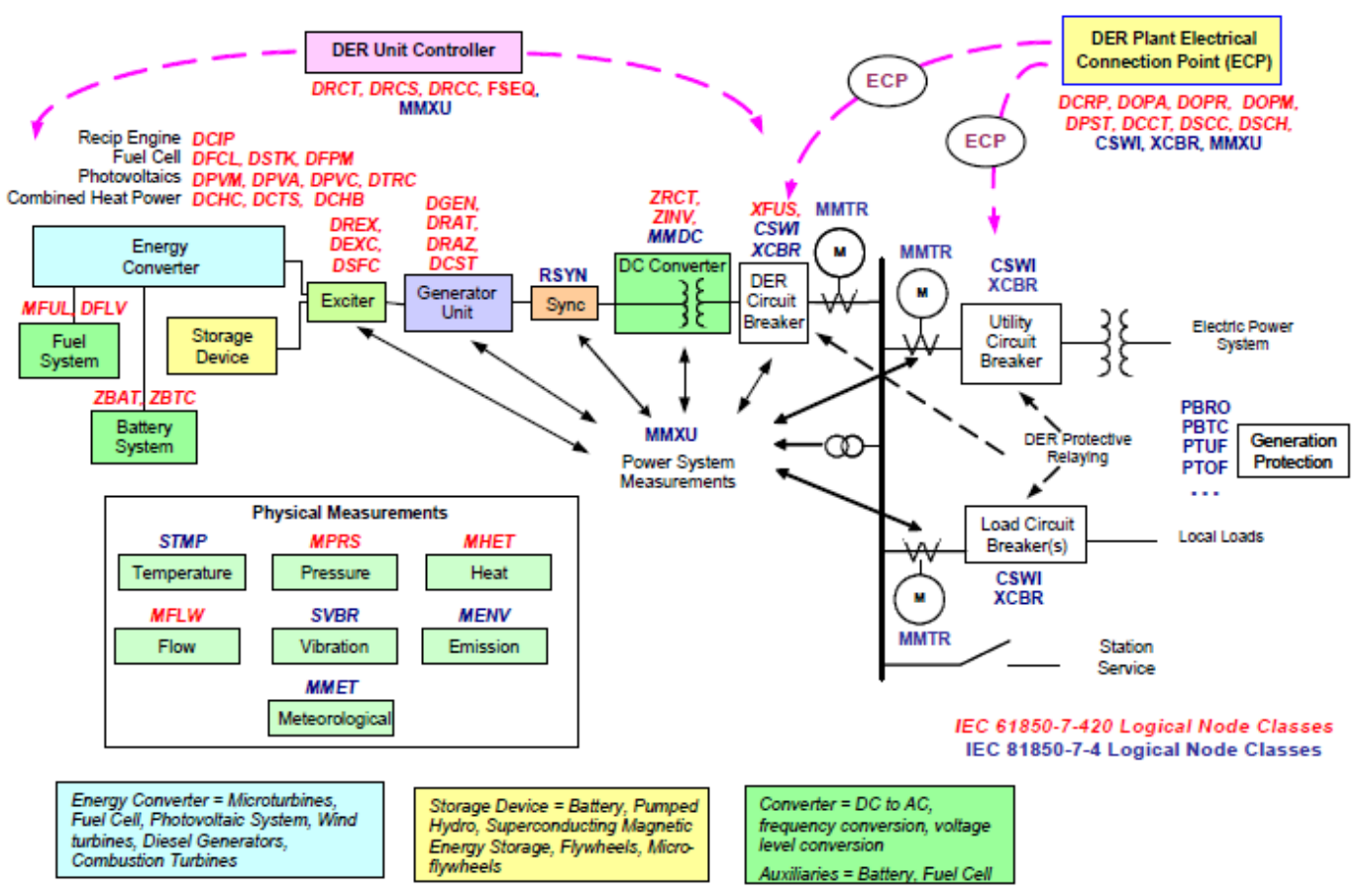

Figure 6. Overview of LDs and LNs for DERs in IEC 61850-7-420 [22]

Microgrids can be modeled by using the predefined LNs in the above mention standards and/or by defining new logical nodes (if missing in the standard) in-accordance to the guidelines of IEC. A list of newly added logical nodes can be seen in [23]. Figure 6 depicts an overview of the LDs and LNs defined in IEC 61850-7-420 for DERs.

\subsection{Realization of Proposed Protection Scheme Through IEC 61850}

An IEC 61850-based framework has been proposed for realization of the proposed hybrid adaptive protection scheme. In order to assure the interoperability among the developed DERs, a gateway has been used for exchanging data with IEC 61850-based devices similar to [21]. Figure 7 depicts the overall model of the proposed hybrid adaptive protection scheme according to IEC 61850 standard. The station level devices are responsible for the functions of central controller as defined in previous sections. Each of the process level IED is responsible for functioning as a local controller. Each local controller can communicate with its devices through the proposed gateway. The bay level IEDs are responsible carrying out the functions of merging units and intelligent input/output (IO) units.

The information system of a microgrid can be developed by using the process level, bay level and station level devices as proposed in IEC 61850 standard [23]. The process level information models and LNs for microgrids are available in IEC 61850-7- 420 as mentioned earlier. The bay level is mainly composed of IEDs. The major tasks of the bay level IEDs are to receive and analyze the measured values and status information from the process level devices and inform the station level units. In addition, these IEDs are also responsible for receiving the control and setting commands from station level, and transfer them to the process level devices. Microgrid control system could be categorized as the station level. The major tasks of the station level control units of a microgrid are operation monitoring, resource scheduling and optimization, planning and management, and stability control [23]. 


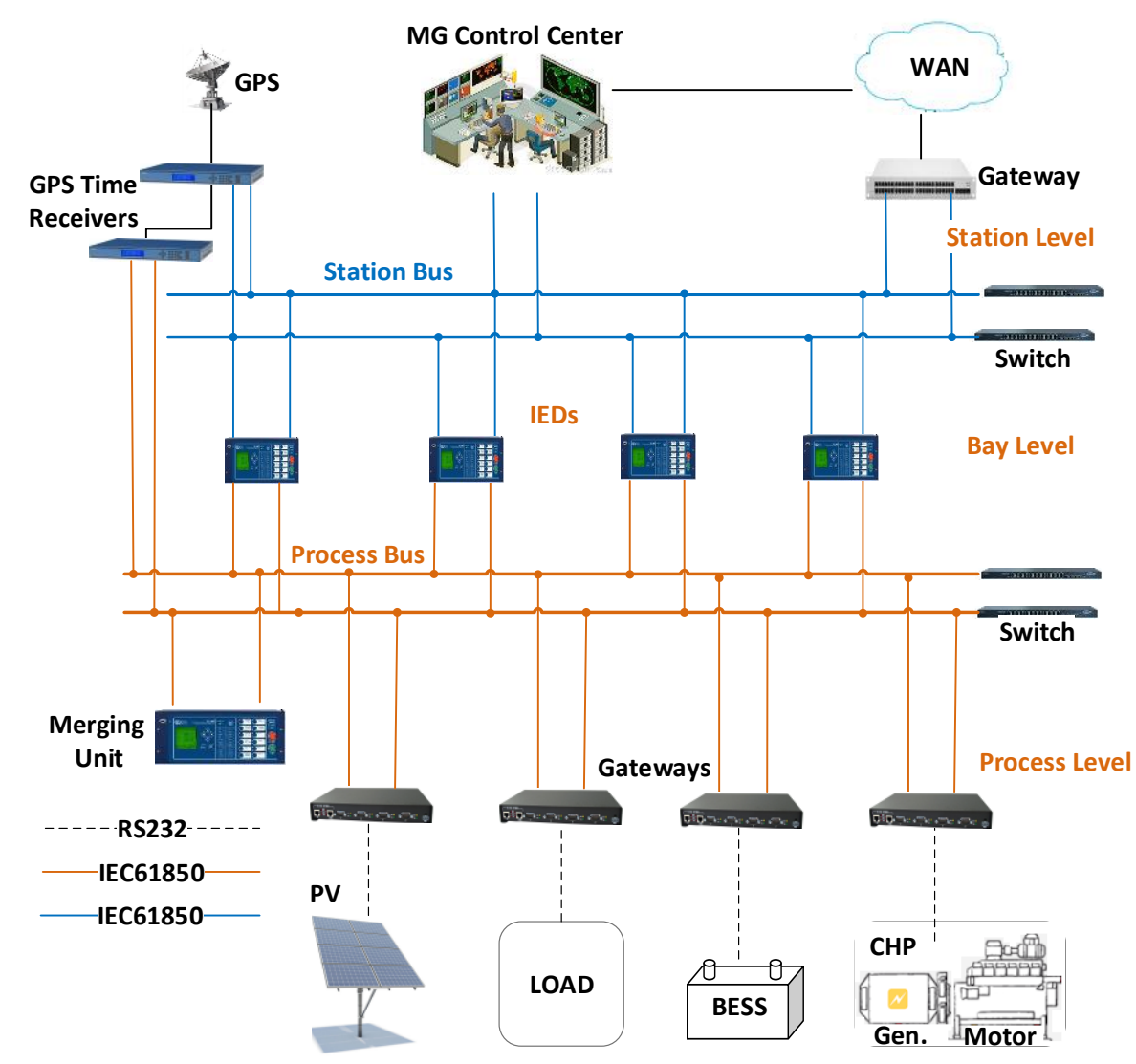

Figure 7. Architecture for Implementation of Proposed Hybrid Adaptive Protection Scheme Through IEC 61850-Based IEDs [6].

Fast-speed messages (trip commands and lock instructions) and original data messages (sampled current and voltages values) are exchanged between the IEC 61850 interface of the gateway and the process bus. The responsible IEDs subscribe to the GOOSE messages and SVs, which are published by the intelligent input/output (IO) units and MUs respectively. Low or medium-speed messages are exchanged between the bay and station level devices. These messages could be automation control and power operation data, protection events and setting values, and time synchronization messages by using the manufacturing message specification (MMS) protocol.

Figure 8 shows the flowchart for implementation of the proposed hybrid adaptive protection scheme through IEC 61850-based IEDs. The functions of local controllers, central controller, gateway, IEDs, and MUs are as follows.

- All the configuration files will be upload to the respective devices (IEDs) in the process, bay, and station level devices by the station level controller.

- The central controller will monitor all the local controllers. If any anomaly is detected by the central controller or reported by a local controller, central controller will send information to all the local controller via bay level IEDs.

- Process level IEDs will continuously monitor their respective equipment through GOOSE messages. If any fault is reported by the end-devices to the local controllers, process level IEDs will receive the SVs from the MUs and send a trip command to the relay of faulty device through the gateway (between end-device and process bus). 

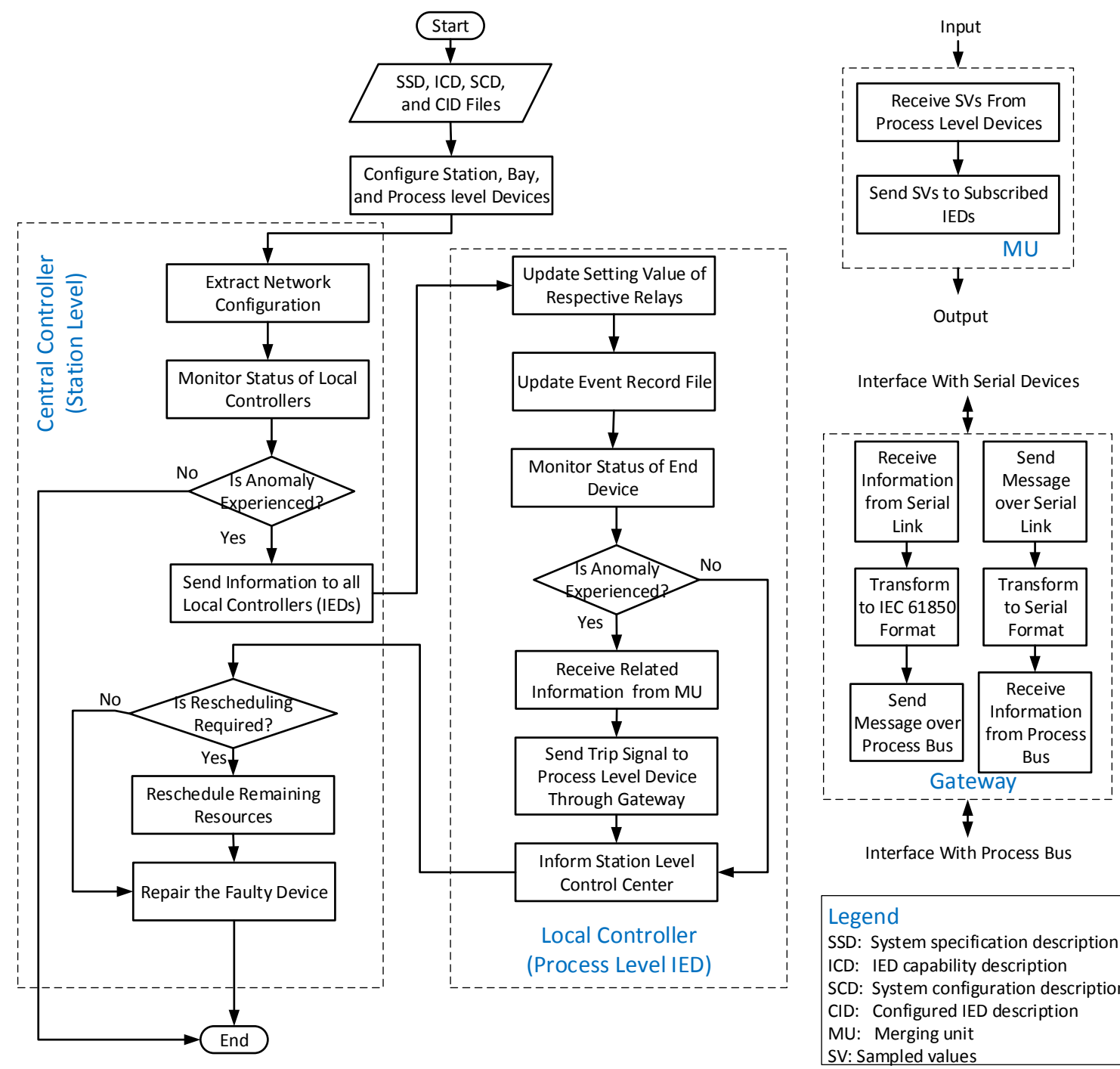

Legend

SSD: System specification description ICD: IED capability description

SCD: System configuration description CID: Configured IED description

MU: Merging unit

SV: Sampled values

\section{Figure 8. Flowchart for Implementation of Proposed Hybrid Adaptive Protection Scheme Through IEC 61850-Based IEDs.}

- The function of the MU is to receive SVs from all the process level IEDs through the process buss and send the SVs to subscribed IEDs periodically. In addition, any IED can request the SVs specific to any event. Figure 8 shows a block diagram for the MU.

- The proposed gateway acts as a master node of the DER control unit over the serial link and acts as an IEC 61850 server for the microgrid monitoring system over the Ethernet link as depicted by Figure 8 .

- After tripping the relay of faulty device, process level IED will inform the station level central controller. The central controller may have to reschedule its resources.

- The faulty equipment will be replaced with a healthy equipment or will be plugged in again after repairing the faulty equipment.

- In this way, the proposed hybrid adaptive protection scheme can be realized through IED 61850. The computational burden and data storing is distributed among local controllers and the central controllers.

\section{Conclusion}

A hybrid protection scheme for adaptive protection of microgrids has been proposed in this paper. The proposed hybrid protection scheme exploits the merits of both centralized 
and decentralized adaptive protection schemes. Computational burden is shared among different level devices and data storage is distributed. Due to this layered processing of data and distributed storage of data, the proposed hybrid adaptive protection scheme is well suited for realization through IEC 61850-based IEDs. In order to assure interoperability between the end devices and IEC 61850-process bus, a gateway has been proposed for transforming serial link data to IEC 61850 standard format. A framework for realization of the proposed hybrid adaptive protection scheme through IEC 61850-based IEDs is also formulated.

\section{Acknowledgement}

This work was supported by Incheon National University Research Grant in 2014.

\section{References}

[1] A. Saima, Y. Simmhan and V. K. Prasanna, "Improving energy use forecast for campus micro-grids using indirect indicators", In: IEEE 11th International Conference on Data Mining Workshops (ICDMW), Vancouver, Canada, (2011), December 11-14.

[2] R. H. Lasseter, "Microgrids", In: IEEE Power Engineering Society Winter Meeting, New York, USA, (2002), January 27-31.

[3] M. Terry, "Campus microgrids: Opportunities and challenges", In: IEEE Power and Energy Society General Meeting, California, USA, (2012), July 22-26.

[4] D. M. Bui, K.Y. Lien, S.L. Chen, Y.C. Lu, C.M. Chan and Y.R. Chang, "Investigate dynamic and transient characteristics of microgrid operation and develop a fast-scalable-adaptable algorithm for fault protection system", Electric Power Systems Research, vol. 120, (2015), pp. 214-233.

[5] O. Fu, A. Nasiri, A. Solanki, A. Bani-Ahmed, L. Weber and V. Bhavaraju, "Microgrids: Architectures, controls, protection, and demonstration", Electric Power Components and Systems, vol. 43, no. 12, (2015), pp. 1453-1465.

[6] A. Oudalov and A. Fidigatti, "Adaptive network protection in microgrids", International Journal of Distributed Energy Resources, vol. 5, no. 3, (2009), pp. 201-226.

[7] E. Sortomme, S. S. Venkata and J. Mitra, "Microgrid protection using communication-assisted digital relays", IEEE Transactions on Power Delivery, vol. 25, no. 4, (2010), pp. 2789-2796.

[8] N. Hassan and R. H. Lasseter, "Microgrid protection", In: IEEE Power Engineering Society General Meeting, Florida, USA, (2007), June 24-28.

[9] H. Al-Nasseri, M. A. Redfern and F. Li, "A voltage based protection for micro-grids containing power electronic converters", In: IEEE Power Engineering Society General Meeting, Boston, USA, (2006), June 28 - July 2.

[10] S. Voima, K. Kauhaniemi and H. Laaksonen, "Novel protection approach for MV microgrid", In: Proce-edings of the 21st International Conference on Electricity Distribution, Frankfurt, Germany, (2011), June 6-9.

[11] C. J. Seok, A. Hussain, H.S. Kim, J. H. Jang, M.S. Choi and S.J. Lee, "An Improved Method of Fault Indicator Generation Algorithm of FRTU in Distribution Automation System", In: Advanced Power system Automation and protection, Jeju, Korea, (2013), October 28-31.

[12] A. Hussain, M. Aslam and S. M. Arif, "N-version programming-based protection scheme for microgrids: A multi-agent system based approach", Sustainable Energy, Grids and Networks, vol. 6, (2016), pp. 3545 .

[13] T.S. Ustun and R. H. Khan, "Multiterminal hybrid protection of microgrids over wireless communications network", IEEE Transactions on Smart Grid, vol. 6, no. 5, (2015), pp. 2493-2500.

[14] C. Erik, L.W. Wei, H. H. Zeineldin and D. Svetinovic, "A differential sequence component protection scheme for microgrids with inverter-based distributed generators", IEEE Transactions on Smart Grid, vol. 5, no. 1, (2014), pp. 29-37.

[15] T. S. Ustun, C. Ozansoy and A. Zayegh, "Modeling of a centralized microgrid protection system and distributed energy resources according to IEC 61850-7-420", IEEE Transactions on Power Systems, vol. 27, no. 3, (2012), pp. 1560-1567.

[16] H.J. Laaksonen, "Protection principles for future microgrids", IEEE Transactions on Power Electronics, vol. 25, no. 12, (2010), pp. 2910-2918.

[17] A. Oudalov, "New technologies for microgrid protection", In: Proceedings of the Santiago 2013 Symposium on Microgrids, Santiago, Chile, (2013), September 09-12.

[18] A. Hussain, M.S. Choi and S.J. Lee, "A novel algorithm for reducing restoration time in smart distribution systems utilizing reclosing dead time", Journal of Electrical Engineering \& Technology, vol. 9, no. 6, (2014), pp. 1805-1811. 
[19] O. Preiss and A. Wegmann, "Towards a composition model problem based on IEC61850", Journal of Systems and Software, vol. 65, no. 3, (2013), pp.227-236.

[20] ABB review, Special report on IEC 61850, new approach, verification and validation, and case studies, [Available online]: https://library.e.abb.com/public/a56430e1e7c06fdfc12577a00043ab8b/3BSE063756_en_ ABB_Review _Special_Report_IEC_61850.pdf

[21] B.K. Yoo, S.H. Yang, H.S. Yang, W.Y. Kim, Y.S. Jeong, B.M. Han and K.S. Jang, "Communication architecture of the IEC 61850-based micro grid system", Journal of Electrical Engineering and Technology, vol. 6, no. 5, (2011), pp. 605-612.

[22] IEC 61850-7-420, Communication networks and system in power utility automation-Part 7-420: Basic communication structure-Distributed energy resources logical nodes and data objects, [Available online]: http://www.iec.ch., (2011).

[23] D. Wei, W. P. Zi, Q. Shen, Z. Zhao and H. Qu, "Adaptive micro-grid operation based on IEC 61850", Energies, vol. 8, no. 5, (2015), pp. 4455-4475.

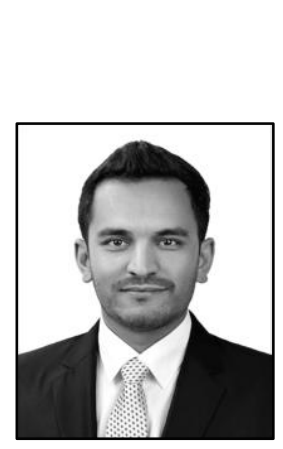

\section{Authors}

Akhtar Hussain received his B.E degree in Telecommunications from National University of Sciences and Technology (NUST) Pakistan in 2011 and M.S in Electrical Power Systems from Department of Electrical \& Electronics Engineering, Myongji University, Korea, in 2014. He worked for SANION, Korea from Jan 2014 to May 2015. Currently he is a Ph.D. student in Power \& Renewable Energy Lab, Department of Electrical Engineering, Incheon National University, Korea. His research interests are power system automation and protection, smart grids, operation of microgrids, and energy management in microgrids.

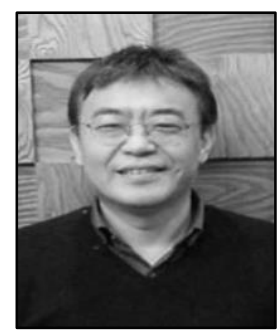

Hak-Man Kim received his first Ph.D. degree in Electrical Engineering from Sungkyunkwan University, Korea in 1998 and received his second $\mathrm{Ph}$. D. degree in Information Sciences from Tohoku University, Japan, in 2011, respectively. He worked for Korea Electrotechnology Research Institute (KERI), Korea from Oct. 1996 to Feb. 2008. Currently, he is a professor in the Department of Electrical Engineering, Incheon National University, Korea. His research interests include microgrid operation \& control and DC power systems. 